\title{
Left trisectionectomy combined with resection of the right hepatic vein and inferior vena cava after right hepatic vein embolization for advanced intrahepatic cholangiocarcinoma
}

\author{
Toshihiro Suzuki, Tomoki Ebata, Yukihiro Yokoyama, Takashi Mizuno, Tsuyoshi Igami, Junpei Yamaguchi, \\ Shunsuke Onoe, Nobuyuki Watanabe and Masato Nagino*
}

\begin{abstract}
Background: When the inferior right hepatic vein (IRHV) is present, left hepatic trisectionectomy with resection of the right hepatic vein (RHV) is theoretically possible without reconstruction of the RHV. We here report a successful case of this extended hepatectomy after RHV embolization for advanced intrahepatic cholangiocarcinoma.

Case presentation: A 71-year-old man was admitted to our clinic with abdominal pain. Computed tomography showed a cholangiocarcinoma located at the caudate lobe that involved the inferior vena cava (IVC) and the roots of the three major hepatic veins. Portal vein embolization of the left and right anterior portal veins was performed. As the IRHV was present but thin, RHV was also embolized. Left hepatic trisectionectomy with resection of the involved IVC and RHV, preserving the IRHV, was done. The IVC was reconstructed with artificial graft. The patient was discharged on postoperative day 36.
\end{abstract}

Conclusion: RHV embolization is useful in extended left trisectionectomy with resection of the RHV when the IRHV is present but thin.

Keywords: Right hepatic vein embolization, Left hepatic trisectionectomy, Intrahepatic cholangiocarcinoma

\section{Background}

When the inferior right hepatic vein (IRHV) is present, left hepatic trisectionectomy with resection of the right hepatic vein (RHV) is theoretically possible without reconstruction of the RHV. When the IRHV is "thin," clinical utility of RHV embolization was reported [1, 2]. We here report a successful case of left hepatic trisectionectomy combined with resection of the RHV and inferior vena cava (IVC), preserving the IRHV, after RHV embolization for advanced intrahepatic cholangiocarcinoma.

\footnotetext{
* Correspondence: nagino@med.nagoya-u.ac.jp

Division of Surgical Oncology, Department of Surgery, Nagoya University Graduate School of Medicine, 65 Tsurumai-cho, Showa-ku, Nagoya 466-8550, Japan
}

\section{Case presentation}

A 71-year-old man was admitted to our clinic with abdominal pain. Contrast-enhanced computed tomography $(\mathrm{CT})$ showed a tumor located at the caudate lobe that involved the IVC and the roots of the three major hepatic veins (Fig. 1a, b). The diagnosis of an advanced intrahepatic cholangiocarcinoma was made. Neither lymph node metastasis nor distant metastasis was detected. He had no jaundice and was in good general condition.

The only possible procedure to achieve curative resection was a left hepatic trisectionectomy combined with resection of the IVC and the three major hepatic veins. The volume of the right posterior sector was $333 \mathrm{~cm}^{3}$ (32.3\% of the whole liver). The plasma disappearance rate of indocyanine green was 0.154. Portal vein 

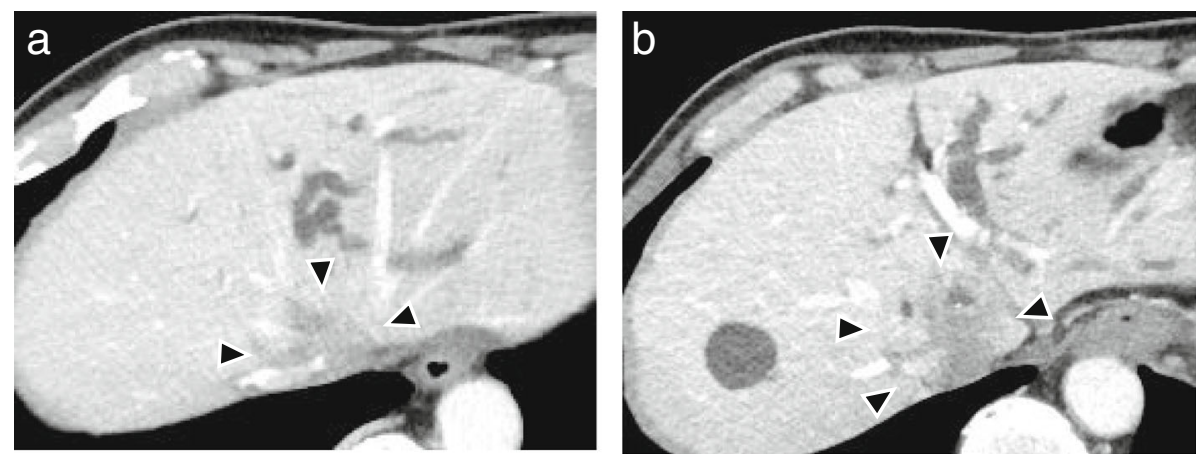

Fig. 1 a Computed tomography showed an irregular tumor (arrowheads) involving the inferior vena cava and the root of the three major hepatic veins. $\mathbf{b}$ Left hepatic bile duct was dilated due to tumor invasion

embolization (PVE) of the left and right anterior portal veins was performed to increase the volume of the right posterior sector. In addition, as this case had a "thin" IRHV, embolization of the RHV was planned, with the aim of simplifying the surgical procedure by preserving the IRHV. Seven days after the PVE, the RHV was embolized using an Amplatzer vascular plug- $\mathrm{II}^{\odot}$ (St. Jude Medical, St. Paul, Minnesota, USA), which was expected to develop collaterals from the RHV to the IRHV (Fig. 2a, b). To assess the feasibility of RHV resection, we ensured collaterals to the IRHV under balloon occlusion of the RHV. A CT scan obtained 29 days after the RHV embolization demonstrated that the volume of the right posterior sector had increased up to $562 \mathrm{~cm}^{3}(42.9 \%$ of the whole liver) and that the diameter of the IRHV had enlarged to $7.7 \mathrm{~mm}$, from $3.5 \mathrm{~mm}$ before embolization (Fig. 3a, b).

Surgery was performed 35 days after the RHV embolization. A left hepatic trisectionectomy with partial resection of the caudate lobe was performed. The involved IVC and RHV were also resected en bloc, and the IRHV was preserved as planned. Before the involved
IVC was resected, we placed the temporal venousvenous bypass between the IVC distal to the renal veins and the right atrium. The resected IVC was reconstructed using a polytetrafluoroethylene (PTFE) vascular graft (Fig. 4a, b). The operative time was $867 \mathrm{~min}$, and blood loss was 12,428 mL.

Histologically, the tumor was a moderately differentiated adenocarcinoma that had invaded the IVC and all three major hepatic veins and exhibited regional lymph node metastases (Fig. 5). Postoperatively, maximum serum total bilirubin concentration was $5.8 \mathrm{mg} / \mathrm{dl}$ (grade B liver failure). Mild ascites developed, but it was well controlled by diuretics. He was discharged from the hospital in good health on postoperative day 36 and enjoyed an active social life, but he died of recurrence 18 months after the surgery.

\section{Discussion}

Hepatectomy combined with the simultaneous resection of the IVC and three major hepatic veins represents one of the most complicated and challenging procedures in hepatobiliary surgery. An "ex vivo" or "ante situm"
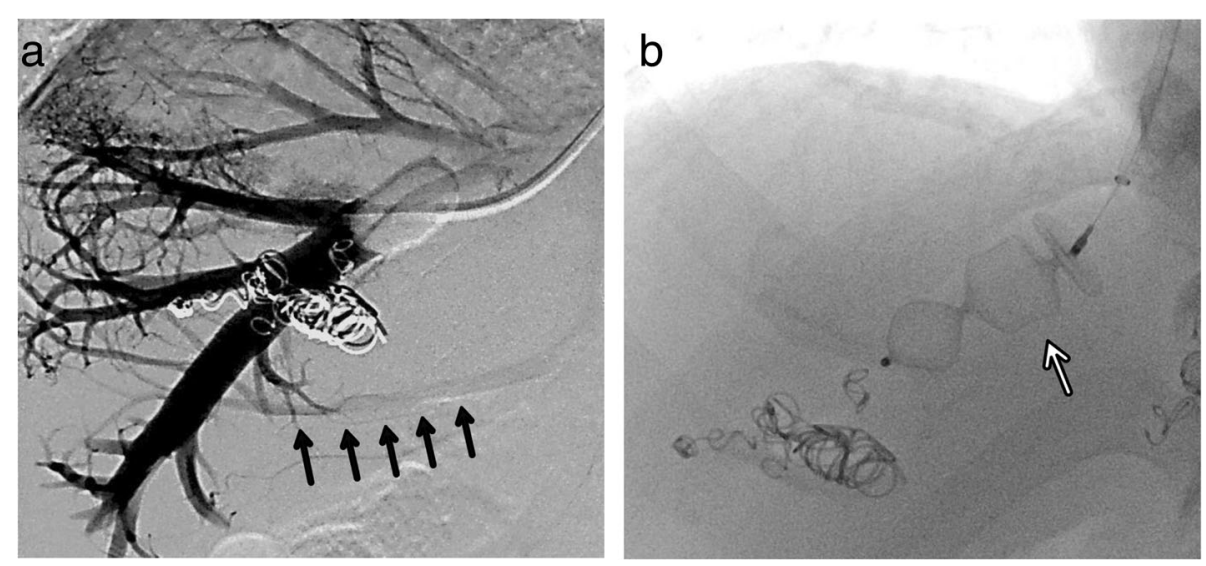

Fig. 2 a Retrograde venography of the right hepatic vein under balloon occlusion showed a "thin" inferior right hepatic vein (arrows). b The right hepatic vein was embolized with an Amplatzer Vascular plug- $\|^{\oplus}$ (white arrow) 

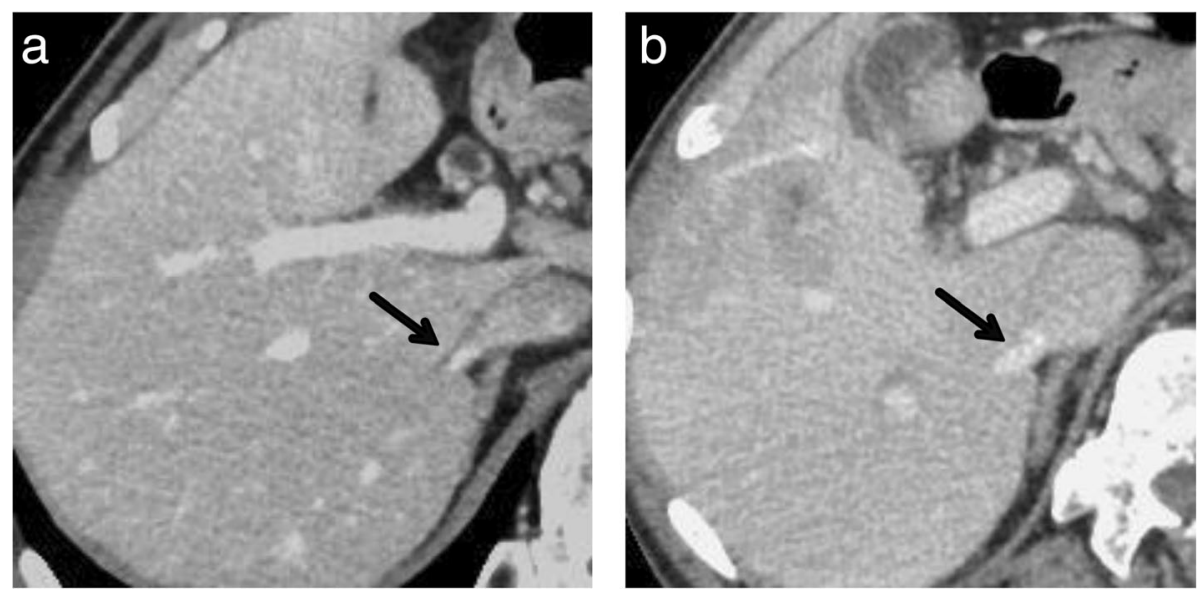

Fig. 3 a Computed tomography obtained before embolization showed that the diameter of the inferior right hepatic vein (arrow) was $3.5 \mathrm{~mm}$. $\mathbf{b}$ The diameter of the vein was enlarged to $7.7 \mathrm{~mm} 29$ days after the right hepatic vein embolization

technique is often required to resect the tumor followed by reconstruction of the IVC and one of the major hepatic veins. However, the presence of the IRHV can circumvent RHV reconstruction even in cases of such an extended hepatectomy. In 1987, Makuuchi et al. mentioned the theoretical feasibility of an IRHVpreserving left hepatic trisectionectomy with resection of the RHV [3]. Thereafter, several authors reported this type of extended hepatectomy in patients having the IRHV $[4,5]$; in these reports, RHV embolization was unnecessary because the IRHV was originally "thick." On the other hand, in patients with a "thin" IRHV like that in the present case, omitting reconstruction of the RHV may be risky due to possible congestion of the remnant liver. To avoid this potential risk, in 2003, one of the present authors $(\mathrm{MN})$ introduced RHV embolization [1], which can help to develop collaterals from the RHV to the IRHV; in that previous case, the tumor involved the RHV but not the IVC; thus, the IVC was not resected. In the present case, the IVC was extensively involved, requiring IVC resection with reconstruction. If the IRHV had been absent, or if the RHV had not been embolized, a curative en bloc resection would have been very difficult.

After RHV embolization, we should wait at least 2 weeks to develop enough collaterals from the RHV to the IRHV. The previous experimental study reported that adequate intrahepatic collaterals develop rapidly after hepatic vein occlusion and are established within 2 weeks in large animals [6].

RHV embolization has a potential risk of coil migration leading to pulmonary embolism. To date, however, no serious procedure-related complications were reported $[7,8]$.
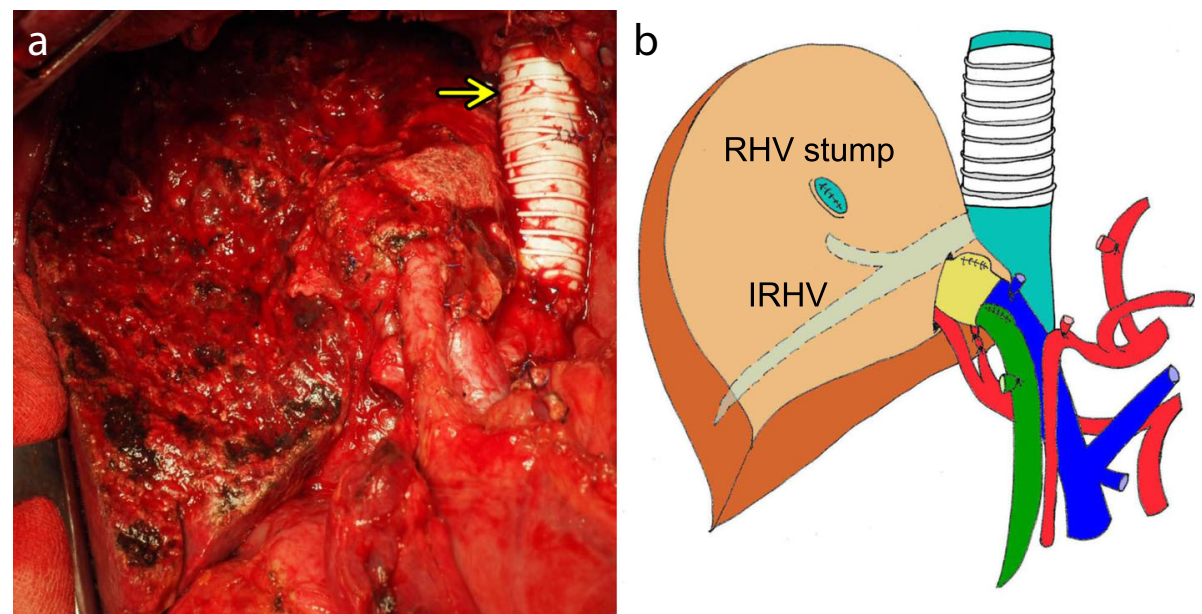

Fig. 4 a Completion photograph after the left hepatic trisectionectomy combined with resection of the inferior vena cava and right hepatic vein. The inferior vena cava was reconstructed with an artificial graft (arrow). b Scheme of the operative view. RHV, right hepatic vein; IRHV, inferior right hepatic vein 


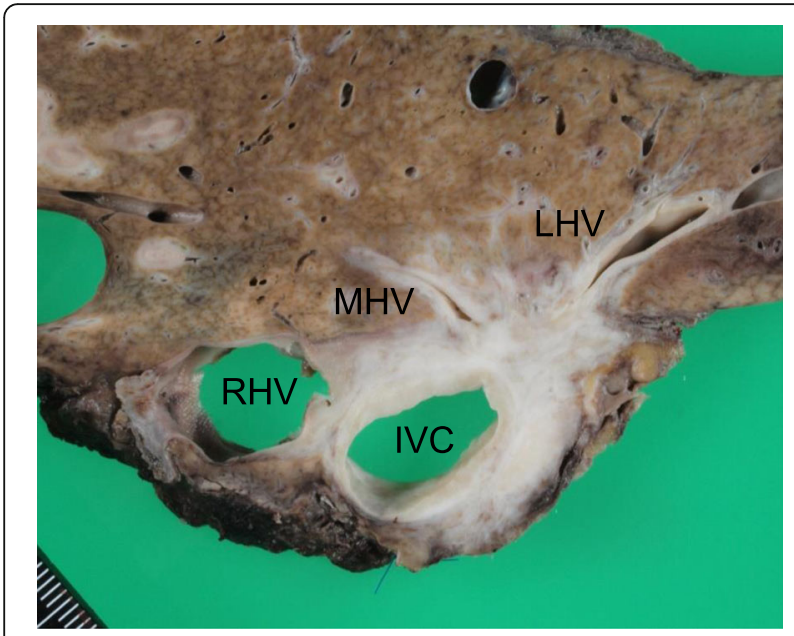

Fig. 5 Cut surface of the resected specimen. RHV, right hepatic vein; MHV, middle hepatic vein; LHV, left hepatic vein; IVC, inferior vena cava

We thought that use of Amplatzer vascular plug could reduce the risk of such migration, providing the plug is oversized by at least $50 \%$.

An important topic of discussion is the definition of "thin" or "thick," which is vague and based on an empirical impression. In other words, to what extent does the diameter of the IRHV guarantee the safety of this extended hepatectomy? Although this issue is still unclear, one previous study classified a large-caliber IRHV as a vein with a diameter of $>18 \mathrm{~mm}$ and a medium-sized IRHV as a vein with a diameter of 5 to 18 $\mathrm{mm}$ [9]. Accordingly, the IRHV in the present case is apparently defined as "thin."

\section{Conclusions}

When a left hepatic trisectionectomy combined with the IVC and the RHV resection is planned, surgeons should focus on the presence or absence of the IRHV and, if it is present but thin, should consider RHV embolization before surgery.

\section{Abbreviations \\ CT: Computed tomography; IRHV: Inferior right hepatic vein; IVC: Inferior vena cava; PTFE: Polytetrafluoroethylene; PVE: Portal vein embolization; RHV: Right hepatic vein}

\section{Funding}

None of the authors have anything to disclose.

\section{Availability of data and materials}

The authors declare that all the data in this article are available within the article.

\section{Authors' contributions}

TS, TE, TM, and MN performed the operation. TI, JY, SO, NW, and YY managed the perioperative course. All authors read and approved the final manuscript.
Ethics approval and consent to participate

This study was carried out in accordance with the principles of the Declaration of Helsinki.

\section{Consent for publication}

Informed consent was obtained from the patient for publication of this case report.

\section{Competing interests}

The authors declare that they have no competing interests.

\section{Publisher's Note}

Springer Nature remains neutral with regard to jurisdictional claims in published maps and institutional affiliations.

Received: 10 April 2019 Accepted: 5 June 2019

Published online: 18 June 2019

\section{References}

1. Nagino M, Yamada T, Kamiya J, Uesaka K, Arai T, Nimura Y. Left hepatic trisegmentectomy with right hepatic vein resection after right hepatic vein embolization. Surgery. 2003;133:580-2.

2. Uji M, Mizuno T, Ebata T, Sugawara G, Igami T, Uehara K, Nagino M. A case of advanced intrahepatic cholangiocarcinoma accidentally, but successfully treated with capecitabine plus oxaliplatin (CAPOX) therapy combined with bevacizumab: a case report. Surg Case Rep. 2016;2:63.

3. Makuuchi M, Hasegawa H, Yamazaki S, Takayasu K. Four new hepatectomy procedures for resection of the right hepatic vein and preservation of the inferior right hepatic vein. Surg Gynecol Obstet. 1987;164:68-72.

4. Baer HU, Dennison AR, Maddern GJ, Blumgart LH. Subtotal hepatectomy: a new procedure based on the inferior right hepatic vein. Br J Surg. 1991;78: $1221-2$.

5. Ozeki Y, Uchiyama T, Katayama M, Sugiyama A, Kokubo M, Matsubara N Extended left hepatic trisegmentectomy with resection of main right hepatic vein and preservation of middle and inferior right hepatic veins. Surgery. 1995;117:715-7.

6. Ku Y, Tominaga M, Sugimoto T, Iwasaki T, Fukumoto T, Takahashi T, Suzuki Y, Kuroda Y. Preoperative hepatic venous embolization for partial hepatectomy combined with segmental resection of major hepatic vein. $\mathrm{Br}$ J Surg. 2002;89:63-9

7. Esposito F, Lim C, Lahat E, Shwaartz C, Eshkenazy R, Salloum C, Azoulay D. Combined hepatic and portal vein embolization as preparation for major hepatectomy: a systematic review. HPB. 2019. https://doi.org/10.1016/j.hpb. 2019.02.023. in press.

8. Hwang S, Ha TY, Ko GY, Kwon DI, Song GW, Jung DH, Kim MH, Lee SK, Lee SG. Preoperative sequential portal and hepatic vein embolization in patients with hepatobiliary malignancy. World J Surg. 2015;39:2990-8.

9. Nakamura S, Tsuzuki T. Surgical anatomy of the hepatic veins and the inferior vena cava. Surg Gynecol Obstet. 1981;152:43-50.

\section{Submit your manuscript to a SpringerOpen ${ }^{\circ}$ journal and benefit from:}

- Convenient online submission

- Rigorous peer review

- Open access: articles freely available online

High visibility within the field

- Retaining the copyright to your article

Submit your next manuscript at $\boldsymbol{\nabla}$ springeropen.com 